\title{
Design of Combined Stationary and Mobile Battery Energy Storage Systems
}

\author{
Hassan S. Hayajneh, Maximiliano Lainfiesta, and Xuewei Zhang
}

\begin{abstract}
To minimize the curtailment of renewable generation and incentivize grid-scale energy storage deployment, a concept of combining stationary and mobile applications of battery energy storage systems built within renewable energy farms is proposed. A simulation-based optimization model is developed to obtain the optimal design parameters such as battery capacity and power ratings by solving a multi-objective optimization problem that aims to maximize the economic profitability, the energy provided for transportation electrification, the demand peak shaving, and the renewable energy utilized. Two applications considered for the stationary energy storage systems are the end-consumer arbitrage and frequency regulation, while the mobile application envisions a scenario of a grid-independent battery-powered electric vehicle charging station network. The charging stations receive supplies from the energy storage system that absorbs renewable energy, contributing to a sustained DC demand that helps with revenues. Representative results are presented for two operation modes and different sets of weights assigned to the objectives. Substantial improvement in the profitability of combined applications over single stationary applications is shown. Pareto frontier of a reduced dimensional problem is obtained to show the trade-off between design objectives. This work could pave the road for future implementations of the new form of energy storage systems.
\end{abstract}

Index Terms-Battery energy storage systems, multi-objective optimization, renewable energy, electric vehicle charging station.

\section{INTRODUCTION}

$\mathrm{B}$ ATTERY energy storage systems (BESSs) have been deployed to meet the challenges from the variability and intermittency of the power generation from renewable energy sources (RESs) [1]-[3]. Without BESS, the utility grid (UG) operator would have to significantly curtail renewable energy generation to maintain system reliability and stability [4], [5]. In the case of wind energy, examples of non-fully utilized wind farms are provided in [6]-[8]. Figure 1 compares the actual grid power injection from a $250 \mathrm{MW}$ wind farm (Chapman Ranch, Texas) in 2018 with the calculated power generation capability. The wind farm averaged an actual power production of about $17.14 \mathrm{MW}$ ( $\sim 7 \%$ of its capacity). Using the weather conditions in 2018 for the same area (acquired from [9]), and assuming the wind turbines with a $25 \%$ efficiency factor (EF), the calculated power production without curtailment could have been 3 4 times higher than the actual power injection. The difference

H. S. Hayajneh is with Texas A\&M University-Kingsville, Kingsville, TX 78363 USA (e-mail: hassan.hayajneh@tamuk.edu).

X. Zhang is with Texas A\&M University-Kingsville, Kingsville, TX 78363 USA (e-mail: xuewei.zhang@tamuk.edu). between the two profiles forms the potential wasted power (average to average) that could have been stored in energy storage systems for other applications.

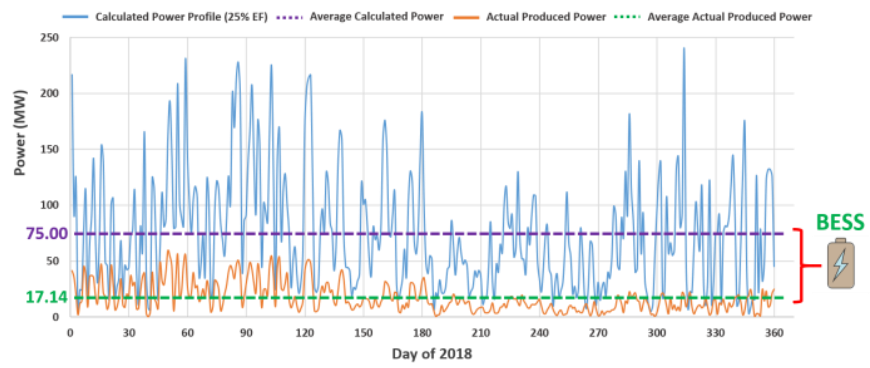

Fig. 1. Comparison between actual produced power profile of $250 \mathrm{MW}$ wind farm in Texas, USA in 2018 and calculated power profile assuming $25 \%$ efficiency factor for wind turbines.

Grid-scale, stationary BESSs have had multiple conventional applications such as (i) end-consumer arbitrage (ECA) [10], [11] to enable consumers to take advantage of lower energy prices due to BESS, (ii) resource adequacy and reserve capacity [12]-[14] ensuring the safe and reliable operation of the UG in real-time by providing sufficient resources, (iii) frequency regulation (FR) [15], [16] to maintain the $\mathrm{AC}$ frequency within tight tolerance bounds and increase the grid stability, (iv) voltage support [17] to absorb or deliver reactive power and serve to keep a specific voltage level on the grid, (v) black-start [18], [19] to restore power plant's electricity or portions of electric grid to operation after a total or partial shutdown, and (vi) transmission congestion relief [20], [21] to enhance the transmission network capacity when the capability of demand for transmission is surpassed. BESSs have gained increasing popularity in the latest decades. According to the U.S. Energy Information Administration (EIA), at the beginning of 2018, the large-scale battery storage in the U.S. was accounted for 708 MW of powered capacity and almost 900 MWh of energy capacity [22]. Worldwide, the installed capacity of BESS is predicted to rise by nearly $20 \mathrm{GW}$ per year [23]. However, the main bottleneck limiting the BESS deployment remains the fairly high costs of the storage technology [24].

In two previous studies [6], [25], driven by data records which show that a wind farm supplies energy to the UG at levels much lower than its real-time capacity (Figure 1), and inspired by [26]

M. Lainfiesta is with the Rocky Mountain Institute, Boulder, CO 80301 USA, (e-mail: mlainfiesta@rmi.com). 
which demonstrated improvements of the investment attractiveness for stationary BESSs by combining applications, it was proposed that the BESS profitability can be significantly enhanced if part of it can be deployed as mobile energy sources to provide backup power to critical facilities or for emergencies, to deliver continuous power supply during grid maintenance and repair, and more interestingly, to meet the energy demand of electric vehicle charging station (EVCS) networks.

The transportation sector witnessed a dramatic growth in the share of electric vehicles (EVs) which in return helps to mitigate the disastrous effects of greenhouse gas (GHG) emissions on the planet, however, this growth will also cause serious issues (e.g., power congestion) to the UG when about one-sixth of the vehicles on the road go electric over the next decade [27], [28]. As an alternative solution to the reinforcement of the electric grid, one may consider the deployment of grid-independent battery-powered EVCS network designs, with the large-scale batteries (MWh) shipped back and forth between EVCSs and BESS plant by fleets of electric truck. From the BESS plant (supplier) point of view, this would account for a continuous DC demand, in contrast to the grid services (AC demand) that are generally intermittent and temporary. With more battery utilization and a guaranteed higher revenue in return, the mobile BESS concept (as illustrated in Figure 2) holds the promise to become a sustainable business model. The proposed BESS stores the extra available power from the RES farms and uses it in several applications: stationary applications like endconsumer arbitrage and frequency regulation (AC demands), and mobile application (DC demand, here meeting demands of the electric vehicle charging stations). The arrows indicate the directions of flow of energy. The conceptual BESS model and the proposed applications are shown in Figure 3.

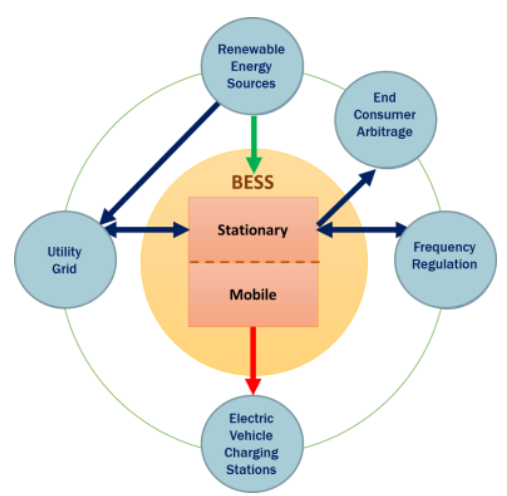

Fig. 2. Diagram of the proposed BESS.

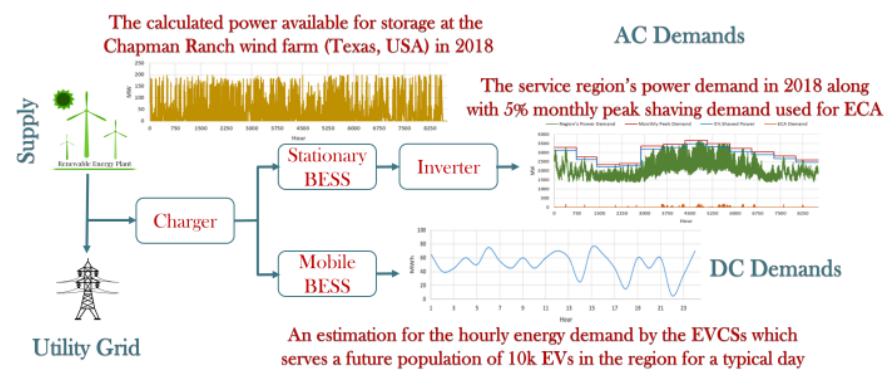

Fig. 3. Diagram of the conceptual BESS model with the proposed applications. Better resolutions for the embedded figures are available in Figure 4.
There are two limitations of [6]: firstly, due to the unavailability of real data, algorithms were used to generate time series of load and portions of wind power production; secondly, only two BESSs with specific capacities were considered, i.e., no optimal design. To address these problems, this work develops a data-driven, simulation-based optimization model to enlighten the planning of a BESS next to RES such as a wind farm. The model can generate key design parameters such as the capacity and power rating by solving a multi-objective optimization problem that aims to maximize the economic profitability index (PI), the energy provided into transportation electrification, the load peak shaving, and the amount of wind energy stored. We consider two operation modes (DC priority and AC priority) and different sets of weights assigned to the above objectives. The results confirm the substantial increase in the profitability of the combined stationary and mobile applications over only stationary applications. This work is intended to help real-world scenarios and future demonstration projects.

The rest of the paper is organized as follows. In Section II, the system configuration, data sources, and model formulation are described. The main results and discussions are presented in Section III. Some conclusions and suggestions for future work can be found in Section IV.

\section{PROBLEM DESCRIPTION}

Opposite to the available small-scale technologies of BESS in supporting the electric vehicles charging stations such as battery swapping [29]-[31], hydrogen storage [32], [33], and fuel cells [34], [35], in this work we propose a new framework of large-scale BESS (each battery unit is assumed to be within a capacity of $5 \mathrm{MWh}$ ). The available technologies are not found to be very popular at large scale, in addition to their reliance on the utility grid as the primary feeder of energy to the EVCSs. Part of the BESS we are proposing here is movable (mobile) and used to power grid-independent EVCS networks, as shown in Figures 2 and 3. The remaining part of the BESS is for conventional, stationary applications, i.e., ancillary services to the UG. The focus is to develop a data-driven, simulation-based optimization framework to obtain the optimal parameters of the proposed BESS. In this section, the data inputs of the model will be described first, then the modules of the simulation model will be introduced, and finally, some technical details of the optimization process will be provided.

\section{A. Data Inputs}

The data is collected from an operating RES, the Chapman Ranch wind farm (Texas, USA) with an installed generation capacity of $250 \mathrm{MW}$, including the actual hourly weather data (wind speed, air density, etc.) for the same region [9]. Using the same method used in [6], the hourly profile of the real-time wind power generation capacity in 2018 is calculated. Then deducting the actual power injected into the utility grid from the wind farm (data source: ERCOT) to estimate the amount of energy available for storage, which is plotted in Figure 4(a). This is the upper limit of the wasted power (assuming all wind turbines operational) that would not be injected into the UG if there is no BESS. One objective of the BESS design in this 
work is to maximize the "absorption" of the extra power from the wind farm, thus maximizing the utilization of the RES.

Three BESS applications are considered in this work; two are provided by the stationary portion of the BESS. In [26], among the conventional, stationary applications of BESS, it was shown that, in the scenarios under study, a combination of endconsumer arbitrage as the primary application and frequency regulation as the secondary application results in the highest profitability index. The present work also considers these two applications as the $\mathrm{AC}$ applications (stationary), in addition to the new DC application (mobile).

The first stationary BESS application considered in this study is end-consumer arbitrage, which creates value for potential industrial consumers who may also invest in the project. When the real-time demand of a consumer approaches the monthly peak $\left(D_{\text {peak }}(m)\right)$, the BESS will supply power to shave the peak demands and reduce the demand charges from the utilities. According to the wind farm service region's load profile in 2018 (Figure 4(b), blue curve), and assuming the peak shaving factor is $5 \%$ (i.e., cutting the monthly peak by $5 \%$ ), the profile of the BESS's demand due to ECA application can be calculated, as shown in Figure 4(b) (purple curve).

The other stationary application of BESS is frequency regulation (FR). The annual revenue generated from the frequency regulation application is anticipated as the product of FR capacity reserved in the BESS (in MW) and the service price (in \$/MW-year [36], [37]). Since the available FR event record lacks details and individual FR events are extremely brief and for a short amount of time (10 $\mathrm{s} \sim 1 \mathrm{~min})$, it is excluded from the simulation model which has a time step of 1 hour. Instead, the model treats the FR capacity as a reserved portion of the power ratings of BESS charger/inverter and sets upper and lower bounds of the state-of-charge $(\mathrm{SoC})$ of the BESS that correspond to capacity reserved for FR.

The third application will be met by the mobile portion of the BESS. The demand profile plotted in Figure 4(c) is used. Note that in a previous work [38], for an EVCS network that serves a population of $10,000 \mathrm{EVs}$, the total daily demand is estimated to be $\sim 1200 \mathrm{MWh}$. A random vector with a mean of $50 \mathrm{MWh}$ is generated as the hourly demand profile $D_{E V C S}(h)$. While a more realistic EVCS demand profile can change the numerical details, it would not change the main conclusion that the introduction of the mobile application can improve both the utilization of renewables and the profitability index.

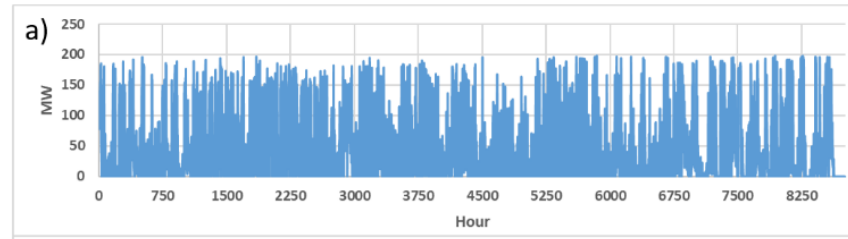

b)

b) - Region's Power Demand - Monthly Peak Demand - $5 \%$ Shaved Power -ECA Demand
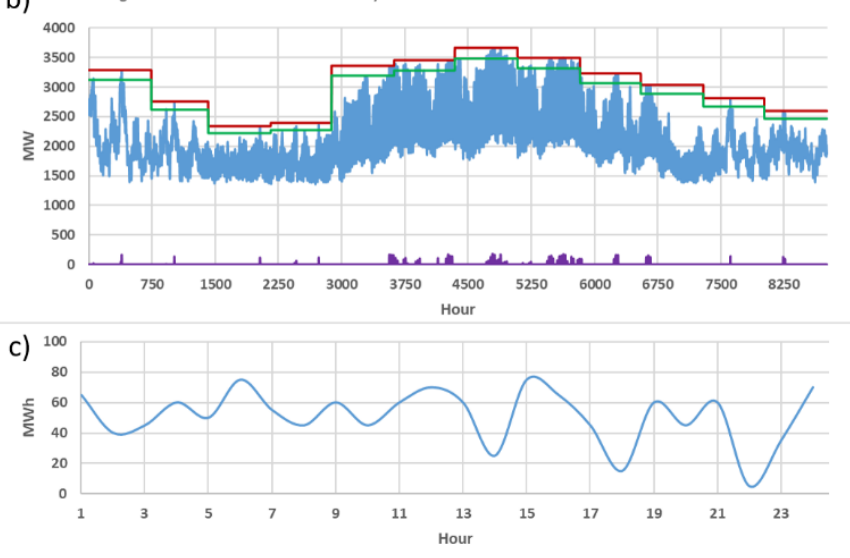

Fig. 4. The simulation model's data inputs. a) The calculated curtailed power available for storage at the Chapman Ranch wind farm (Texas, USA) during 2018 after injecting power to the UG, with the real-time generation estimated based on actual weather data in the region. b) The region's load demand in 2018 along with 5\% monthly peak shaving used for ECA. c) An estimation for the hourly energy demand by the EVCSs which serves a future population of $10 \mathrm{k}$ EVs in the region for a typical day [38].

\section{B. Simulation Modeling}

The simulation model can be run in two modes: The User Mode and the Designer Mode. The User Mode can evaluate the performance indicator (or fitness) of a given BESS, while the Designer Mode can search for the optimal set of parameters that correspond to the highest fitness. Figure 5 presents the block diagram of the simulation model in the User Mode. The model inputs are the data shown in Section II.A, some constants (including physical/financial parameters that are fixed in the simulations, listed in Table I), and the control variables the values of which are specified by the users. As listed in Table II, the model has six control variables (CV) along with their limits. The bounds for all control variables are obtained from the initial solution of optimization process in addition to an assumed optimization safety margin.

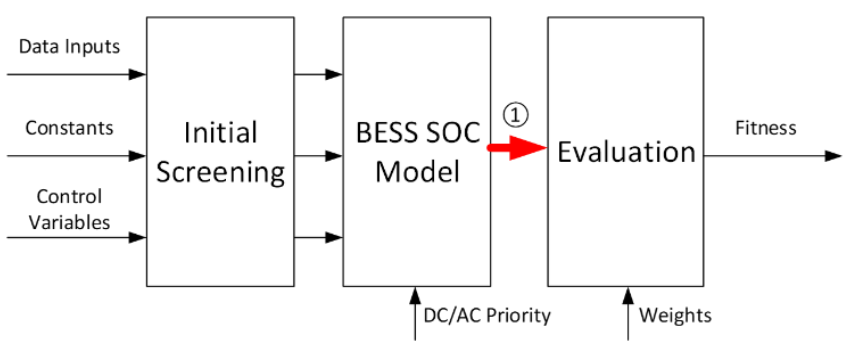

Fig. 5. Block diagram representing the simulation model in the User Mode. The red arrow is explained in the text.

TABLE I. Model constants and their values used in this study.

\begin{tabular}{|l|l|c|}
\hline Symbol & \multicolumn{1}{|c|}{ Meaning and Units } & Value \\
\hline$d c$ & The demand charges $(\$ / \mathrm{MW})$ & $10.3 \mathrm{k}[26]$ \\
\hline
\end{tabular}




\begin{tabular}{|c|c|c|}
\hline$p t$ & Power tariff set by the UG ( $\$ / \mathrm{MWh})$ & 50 \\
\hline$\eta_{\text {charger }}$ & $\begin{array}{l}\text { Efficiency of the Charger }(\%) \text { - used when } \\
\text { calculating the power absorbed from RES into } \\
\text { BESS }\end{array}$ & $95[39]$ \\
\hline$\eta_{\text {inverter }}$ & $\begin{array}{l}\text { Efficiency of the Inverter }(\%)-\text { used when } \\
\text { calculating the power provided into AC } \\
\text { applications }\end{array}$ & $95[39]$ \\
\hline$W A C C$ & Weighted Annual Cost of Capital (\%) & $5.25[26]$ \\
\hline$D C_{\text {rate }}$ & $\begin{array}{l}\text { The price for energy provided to EVCSs } \\
\text { (\$/MWh) [Assumed] }\end{array}$ & 100 \\
\hline$B_{\text {cost }}$ & The cost of the Battery (\$/MWh) & $100 \mathrm{k}$ \\
\hline$C_{\text {cost }}$ & The cost of the Charger $(\$ / M W)$ & $80 \mathrm{k}$ \\
\hline$I_{\text {cost }}$ & The cost of the Inverter $(\$ / M W)$ & $80 \mathrm{k}$ \\
\hline$B_{\text {OMfactor }}$ & $\begin{array}{l}\text { Annual cost of battery operation \& } \\
\text { maintenance as percentage of } B_{\text {cost }}(\%) \\
\text { [Assumed] }\end{array}$ & 5 \\
\hline$C_{\text {OMfactor }}$ & $\begin{array}{l}\text { Annual cost of charger operation \& } \\
\text { maintenance as percentage of } C_{\text {cost }}(\%) \\
\text { [Assumed] }\end{array}$ & 5 \\
\hline$I_{\text {OMfactor }}$ & $\begin{array}{l}\text { Annual cost of inverter operation \& } \\
\text { maintenance as percentage of } I_{\text {cost }}(\%) \\
\text { [Assumed] }\end{array}$ & 5 \\
\hline$F R_{\text {rate }}$ & $\begin{array}{l}\text { Frequency regulation price ( } \$ / \mathrm{MW}-\mathrm{yr}) \text {. Based } \\
\text { on ERCOT } 10.25 \% \text { reserve margin }[40]\end{array}$ & $100 \mathrm{k}$ \\
\hline$P L$ & Project life time (Years) & 20 \\
\hline
\end{tabular}

The first module of the model shown in Figure 5 is called the Initial Screening, which examines the compatibility within the model inputs and the feasibility of other parameter settings. Among the screening criteria, $C V_{2}$ should be lower than $C V_{1}$; $C V_{5}$ should never exceed the value of $C V_{3}$ or $C V_{4}$; along with other constraints to be detailed below. As a model assumption, the reserved SoC for FR in the BESS is equal to the FR reserved power multiplying by $\delta=1 / 6$ hour, which is mandated to be below half of the size of the stationary portion of the BESS $\left(C V_{1}-C V_{2}\right)$. Note that this module will be disabled during the optimization process in the Designer Mode since the criteria are included as constraints there; Initial Screening is unnecessary.

TABLE II. Control variables and their bounds.

\begin{tabular}{|c|l|l|c|c|}
\hline CV & Symbol & \multicolumn{1}{|c|}{ Meaning and Units } & $\begin{array}{c}\text { Lower } \\
\text { bound }\end{array}$ & $\begin{array}{c}\text { Upper } \\
\text { bound }\end{array}$ \\
\hline$C V_{1}$ & $B E S S_{\text {size }}$ & $\begin{array}{l}\text { The total size of the BESS } \\
\text { (MWh) }\end{array}$ & 350 & 2000 \\
\hline$C V_{2}$ & $B E S S_{\text {mobile }}$ & $\begin{array}{l}\text { The size of the mobile } \\
\text { portion of the BESS } \\
\text { (MWh) }\end{array}$ & 50 & 500 \\
\hline$C V_{3}$ & $P_{\text {charger }}$ & $\begin{array}{l}\text { The power rating of the } \\
\text { charger (MW) }\end{array}$ & 250 & 600 \\
\hline$C V_{4}$ & $P_{\text {inverter }}$ & $\begin{array}{l}\text { The power rating of the } \\
\text { inverter (MW) }\end{array}$ & 250 & 600 \\
\hline$C V_{5}$ & $F R_{\text {reserve }}$ & $\begin{array}{l}\text { The power reserved for } \\
\text { frequency regulation (FR) } \\
\text { application (MW) }\end{array}$ & 10 & 100 \\
\hline$C V_{6}$ & $S F$ & $\begin{array}{l}\text { The shaving factor of peak } \\
\text { demand (\%) }\end{array}$ & 0.5 & 15 \\
\hline
\end{tabular}

After the control variables pass the initial screening, all the inputs are passed on to the BESS SoC model. The BESS SoC model is simulated hour-by-hour for a year to make decisions on power absorption from the RES and how the demands are met. There are two operational modes of the proposed BESS, namely DC priority and AC priority. Here DC/AC priority means that if at the current hour, the available energy supply (wind farm extra and BESS combined) cannot meet $100 \%$ of the combined AC (stationary applications) and DC (mobile applications) demands, the system will try to meet the DC/AC demand first. The BESS SoC dynamics follows previous work [25]. There are lower and upper bounds of $\mathrm{SoC}$ as required by the FR reserve. Within this range, the $\mathrm{SoC}$ will be updated hourly by the difference between supply and demand. The only complication in the model is that the actual mobile portion size is time-dependent since every hour fully-charged batteries are shipped out to EVCSs and drained batteries are transported back by trucks dispatched out an hour earlier.

There are four hourly profiles as the output of the model: (a) $P_{S}$, the power produced by the fully operational RES less the amount injected to the UG, (b) $P_{A}$, the actual power absorbed by the BESS from the RES, (c) $P_{E C A}$, the actual power supplied for peak shaving (ECA), and (d) $P_{E V C S}$, the actual power provided for mobile applications (EVCSs). These data will be diverted to the Evaluation module to generate the BESS performance indices.

There are four normalized indices between 0 and 1, and the higher the value of each index, the better.

(i) Index $1\left(f_{1}\right)$, the investment attractiveness (IA): is defined as the ratio of the number of years the PI being above 1 to the project lifetime (20 years in this study). Conceptually, it includes the reduction of demand charges and the corresponding energy charge (ECA), FR revenue, and net profits from the mobile application of the BESS. For the latter, $\$ 100 / \mathrm{MWh}$ is assumed, after deduction of the transportation expenses (truck and labor) from the selling price. Higher values of IA mean more years with net profits. Therefore, the IA index reflects the financial viability of the proposed BESS design.

The calculation of the PI follows:

$$
P I_{y}=\frac{N P V_{y}}{C_{\text {invest }}}
$$

where $C_{\text {invest }}=B_{\text {cost }} B E S S_{\text {size }}+C_{\text {cost }} P_{\text {charger }}+I_{\text {cost }} P_{\text {inverter }}$ stands for the initial capital investment; and $N P V_{y}$, the net present value (\$) up to the end of year $y$, is calculated according to:

$$
N P V_{y}=\sum_{t=0}^{y} \frac{C F_{t}}{(1+W A C C)^{t}}
$$

where $t$ represents the year in which $C F_{t}$ is the cash flow (\$) (the initial investment at $t=0$ is set to be negative; for each subsequent year, the cash flow is the value generated minus the operating expenses), and WACC stands for the weighted average cost of capital. The annual cash flow $(C F)$ that represents the difference between the monetary value generated $(V G)$ by the three BESS applications and the operation and maintenance costs $\left(O M_{\text {cost }}\right)$ for a year is estimated as: 


$$
C F=V G-\sum_{B, C, I} O M_{c o s t}
$$

where $V G$ combines the annual monetary income generated by the energy supplied to EVCSs $\left(V G_{E V C S}\right)$, the capacity reserved for FR $\left(V G_{F R}\right)$, and the energy provided to the ECA $\left(V G_{E C A}\right)$ :

$$
\begin{gathered}
V G=V G_{E V C S}+V G_{F R}+V G_{E C A} \\
V G_{E V C S}=D C_{\text {rate }} \sum_{h=1}^{8760} P_{E V C S}(h) \\
V G_{F R}=F R_{\text {reserve }} F R_{\text {rate }} \\
V G_{E C A}=d c \sum_{m=1}^{12} P_{\text {shaved }}(m)+p t \sum_{h=1}^{8760} P_{E C A}(h)
\end{gathered}
$$

In Eq. (7), $P_{\text {shaved }}$ is each month's actual shaved peak demand that can be obtained by the simulation. And $O M_{\text {cost }}$ in Eq. (3) consists of the contributions from the batteries (B), the chargers (C), and the inverters (I):

$$
\begin{gathered}
\sum_{B, C, I} O M_{\text {cost }}=B_{-} O M_{\text {cost }}+C_{-} O M_{\text {cost }}+I_{-} O M_{\text {cost }} \\
B_{-} O M_{\text {cost }}=B_{\text {OMfactor }} B_{\text {cost }} B E S S_{\text {size }} \\
C_{-} O M_{\text {cost }}=C_{\text {OMfactor }} C_{\text {cost }} P_{\text {charger }} \\
I_{-} O M_{\text {cost }}=I_{\text {OMfactor }} I_{\text {cost }} P_{\text {inverter }}
\end{gathered}
$$

(ii) Index $2\left(f_{2}\right)$ : is the ratio of the actual mobile demand met and the total mobile demand according to the input data.

(iii) Index $3\left(f_{3}\right)$ : is the ratio of the actual peak shaving demand met and the peak shaving demand corresponding to the targeted peak shaving factor. These performance indices (2\&3) show the capability of the BESS to meet the stationary and mobile demands described above. Therefore, they will be specifically considered in the multi-objective optimization in the next section.

(iv) Index $4\left(f_{4}\right)$ : is the ratio of the actual power from the RES stored by the BESS to the total power available for storage according to the input data. Index 4 shows the performance of the BESS in maximizing the utilization of renewables that would be otherwise wasted.

In summary, the calculation of the four indices follows:

$$
\begin{gathered}
f_{1}=\sum_{y=1}^{P L} I_{P I}(y) / P L \\
f_{2}=\min _{h}\left[P_{E V C S}(h) / D_{E V C S}(h)\right] \\
f_{3}=\min _{m}\left(P_{\text {shaved }}(m) /\left[S F \cdot D_{\text {peak }}(m)\right]\right) \\
f_{4}=\min _{h}\left[P_{A}(h) / P_{S}(h)\right]
\end{gathered}
$$

where $I_{P I}(y)=1$ if $P I_{y} \geq 1$ and 0 otherwise. In the cases of PI $<1$ for all years (financial losses), $f_{1}$ can be set to -1 as penalty. The hourly mobile demand $D_{E V C S}(h)$, monthly peak load $D_{\text {peak }}(m)$, and available energy for storage $P_{S}(h)$ are from the input data.

\section{Optimization Method}

In the Designer Mode, the Genetic Algorithm (GA) is used as the solution method to find the optimal values of the control variables that maximize the performance indices in Eqs. (12)(15). The indices are evaluated at each iteration (i.e., each set of control variable values), which runs an hourly simulation of the system for a year. By choosing a vector of weights of the indices $\boldsymbol{w}=\left[w_{1}, w_{2}, w_{3}, w_{4}\right]$, the weighted average of the four indices defines a fitness function $F$, which is also an objective function in the Designer Mode. This work first optimizes $F$ to validate the numerical algorithm and conduct preliminary analysis. Then a multi-objective (focusing on $f_{2}$ and $f_{3}$ ) optimization problem will be formulated and show the pareto frontier. The statement of the single objective optimization problem is:

Maximize $\quad F=\sum_{m=1}^{4} w_{m} f_{m}$

Subject to:

$$
\begin{aligned}
& C V_{2} \geq \max \left(D_{E V C S}\right) \\
& C V_{1}-C V_{2}-2 \delta \cdot C V_{5} \geq 0 \\
& C V_{1}-C V_{3} \geq 0 \\
& C V_{1}-C V_{4} \geq 0 \\
& C V_{3}-C V_{5} \geq \max \left(P_{S}\right) \\
& C V_{4}-C V_{5}-\max \left(D_{\text {peak }}\right) \cdot\left(C V_{6}\right) \geq 0
\end{aligned}
$$

Additionally, Table II provides the bounds for each control variable. The constraints combined are a necessary condition of the operational viability of the BESS.

The simulation model and the optimization processes are implemented in MATLAB. The GA settings are a population size of 500 and 100 iterations for each generation. Figure 6 shows a sample of convergence plot for equal weights.

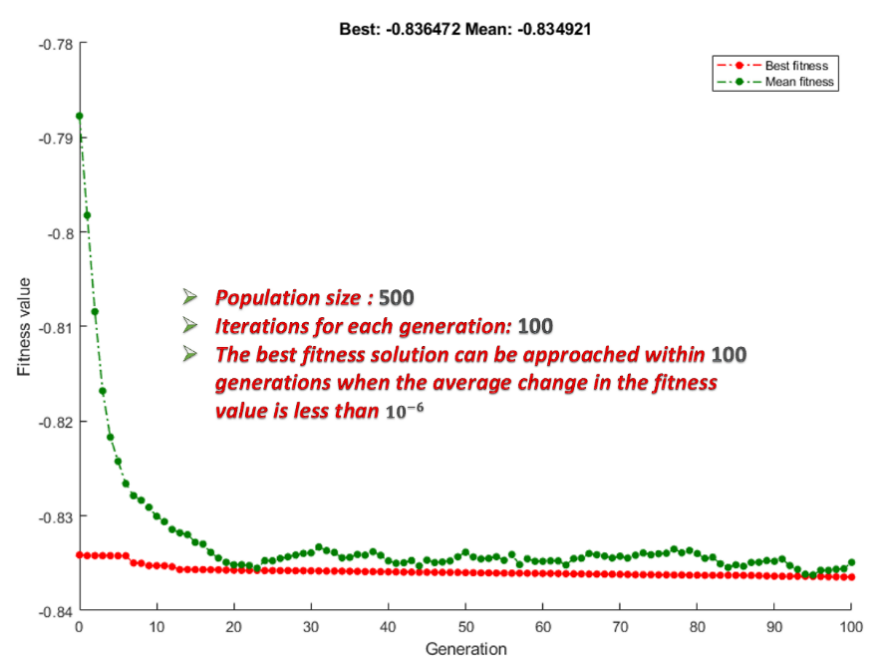

Fig. 6. The convergence plot of GA for the equal weights' scenario $\left(w_{i}=0.25\right)$ and DC priority. In general, the value for the best fitness can be attained within 100 generations.

\section{RESUltS AND DiscuSSION}

\section{A. Optimization of the Weighted Average of Four Indices}

In this subsection, the optimization results of the six control variables in four scenarios are presented. Each scenario had a 
different weight vector. Scenario $1: \boldsymbol{w}=[0.25,0.25,0.25,0.25]$, Scenario 2: $\boldsymbol{w}=[0.3,0.15,0.25,0.3] \quad, \quad$ Scenario 3: $\boldsymbol{w}=$ $[0.5,0.15,0.15,0.2]$, Scenario $4: w=[0.7,0.1,0.1,0.1]$. Under each scenario, results are obtained for the two operation modes: DC priority which indicates supplying the EVCS networks via the mobile BESS, as shown in Figure 7; and AC priority which indicates supplying the ECA demand via the stationary BESS, as shown in Figure 8. One of the key differences between the two modes/cases is that, with more focus on IA (Scenario 4), DC priority leads to a larger mobile BESS size, which is not the case of AC priority. The four performance indices are presented in Figure 9. For the data used, in all cases, Index 4 reaches or is very close to $100 \%$, while there is more actual peak demand shaved in the AC priority mode than the DC priority mode.

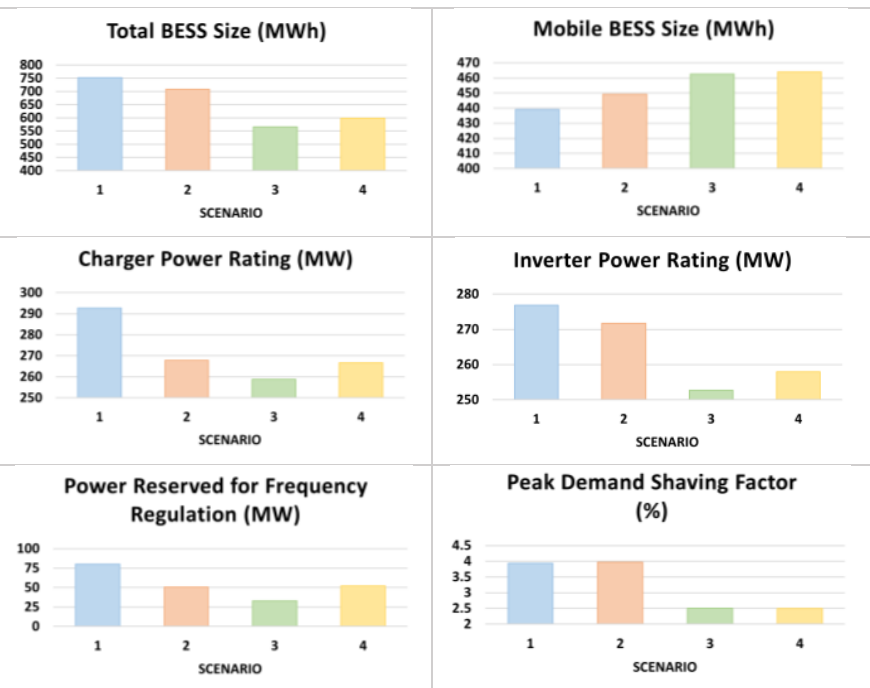

Fig. 7. Optimized sets of the six control variables in four different scenarios (1, $2,3,4)$ under the DC priority operation mode.
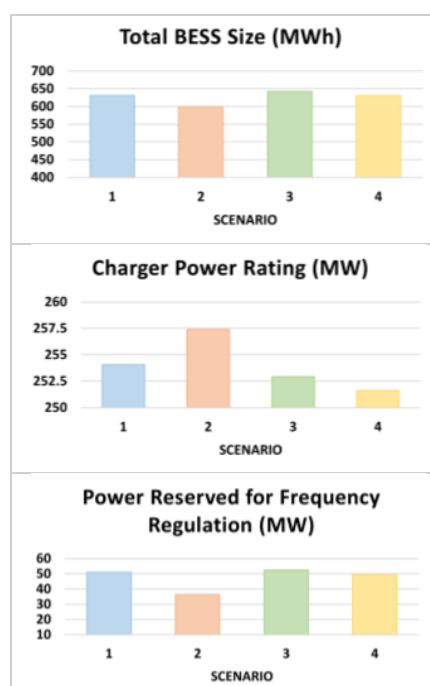

Fig. 8. Optimized sets of the six control variables in four different scenarios (1, $2,3,4)$ under the AC priority operation mode.
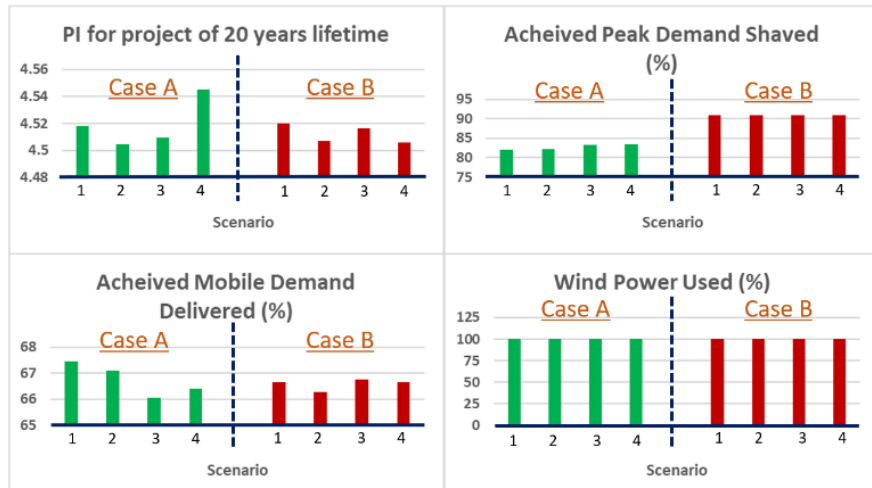

Fig. 9. Four BESS performance indices (PI and Indices 2-4) with the optimized control variables in the four scenarios with the DC priority (case A) and the AC priority (case B).

TABLE III. PIs of different application combinations: 3 applications (EVCS, FR, ECA), 2 applications (FR, ECA), and 1 application (ECA only).

\begin{tabular}{|c|c|c|c|}
\hline \multirow{2}{*}{ Scenario } & \multicolumn{3}{|c|}{ Profitability Index (PI) under the DC Priority } \\
\cline { 2 - 4 } & 3 Applications & 2 Applications & 1 Application \\
\hline $\mathbf{1}$ & 4.5180 & 1.7473 & 0.9388 \\
\hline $\mathbf{2}$ & 4.5048 & 1.5905 & 1.0477 \\
\hline $\mathbf{3}$ & 4.5095 & 1.0059 & 0.5969 \\
\hline $\mathbf{4}$ & 4.5455 & 1.1677 & 0.5416 \\
\hline \multirow{3}{*}{ Scenario } & \multicolumn{3}{|c|}{ Profitability Index (PI) under the AC Priority } \\
\cline { 2 - 4 } & $\mathbf{3}$ Applications & 2 Applications & 1 Application \\
\hline $\mathbf{1}$ & 4.52 & 1.1258 & 0.5278 \\
\hline $\mathbf{2}$ & 4.5069 & 1.0208 & 0.5803 \\
\hline $\mathbf{3}$ & 4.5163 & 1.1337 & 0.5218 \\
\hline $\mathbf{4}$ & 4.5062 & 1.1012 & 0.5214 \\
\hline
\end{tabular}

On average, the PI at the end of the project lifetime is slightly higher under the DC priority than in the AC priority. However, all PIs are in the range of $4.4 \sim 4.6$, which is equivalent to a $7 \sim 8 \%$ annual return over the 20 -year project lifetime. Without a mobile application, we re-ran the model in the User Mode and calculated the PI values at the end of the project lifetime. The results are presented in Table III. If the BESS has only one application (ECA), the PI in most cases is below 1, which means the project cannot break even. Adding frequency regulation as a secondary application approximately doubles the PI, but, in the best case, it only corresponds to an annual return of $3 \%$. It is obvious that the combined stationary and mobile BESS applications, conceptualized in Figures 2 and 3, can increase the profitability and therefore the IA of the largerscale deployment of BESS.

\section{B. Multi-Objective Optimization}

This subsection presents the effort to perform multi-objective optimization of the proposed BESS design. The aim here is to obtain the profile of the Pareto frontier, i.e., the set of optimized solutions that cannot improve an objective without degrading at least one other objective. As mentioned earlier, the focus is on Index 2 and Index 3 , the performance indicators for the DC and $\mathrm{AC}$ demands met by the BESS. Given the overall size of the BESS, it is not difficult to see that the two indices related to the portions of mobile and stationary batteries, which are trending 
in opposite directions and cannot be improved at the same time. It is of practical interests to see the trade-off.

To start with, a reduced dimensional optimization problem needs to be formulated. The linear scalarizing method is used to solve multi-objective optimization via the definition of the following weighted average of the two objective functions:

$$
\text { Maximize } \quad F^{\prime}=w f_{2}+(1-w) f_{3}
$$

where $w$ is a real number between 0 and 1. From the results in the previous subsection, in all cases, the optimum fitness $F$ is reached when the value of the investment attractiveness (IA) equals or exceeds $0.85\left(f_{1} \geq 0.85\right)$ and the value of the actual percentage of wind energy utilized equals $100 \%\left(f_{4}=1\right)$. These will be two additional constraints of the new optimization problem. Now, to obtain the Pareto set of optimum solutions, optimizations are conducted with $w=[0.00,0.05,0.10, \ldots, 1.00]$ of for each operational mode (DC and AC). Figures 10 and 11 present the optimized values for the six control variables under the DC and AC priorities, respectively. Figures 12 and 13 depict the respective Pareto set of optimal solutions.

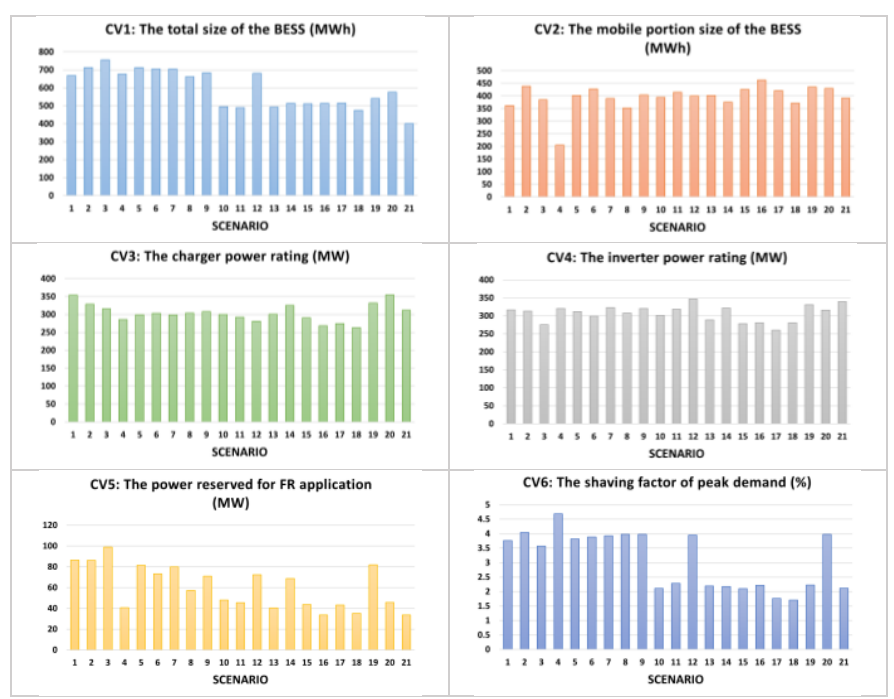

Fig. 10. Optimal solutions of the six control variables with 21 weight factors from 0 (Scenario 1) to 1 (Scenario 21 ) in step of 0.05 under DC priority mode.

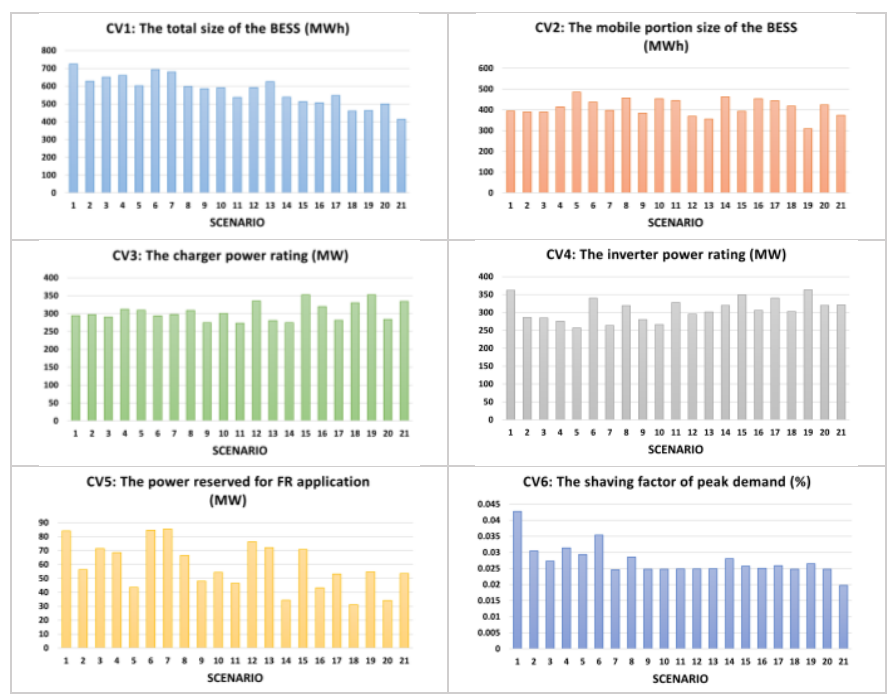

Fig. 11. Optimal solutions of the six control variables with 21 weight factors from 0 (Scenario 1) to 1 (Scenario 21) in step of 0.05 under AC priority mode.

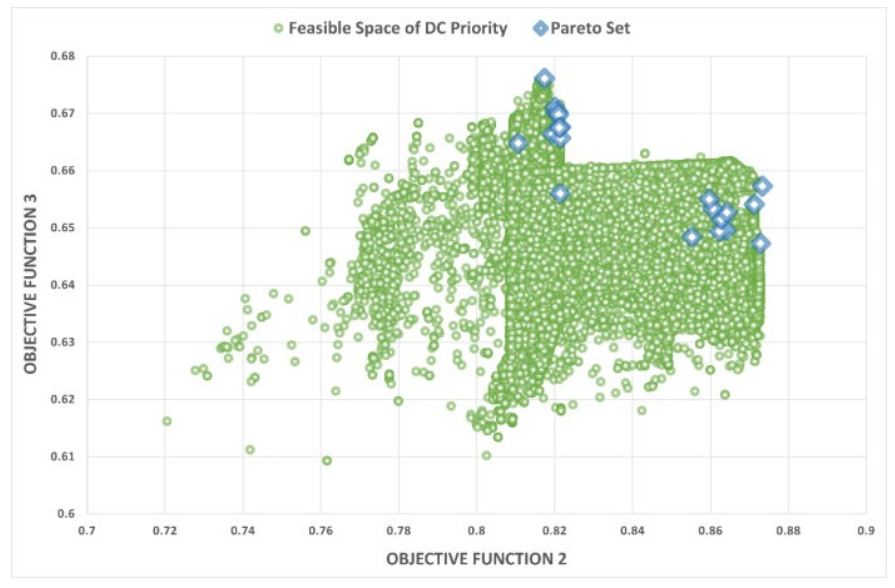

Fig. 12. The profile of feasible solutions under the 21 scenarios and the Pareto set for the two objective functions under the DC priority operation mode.

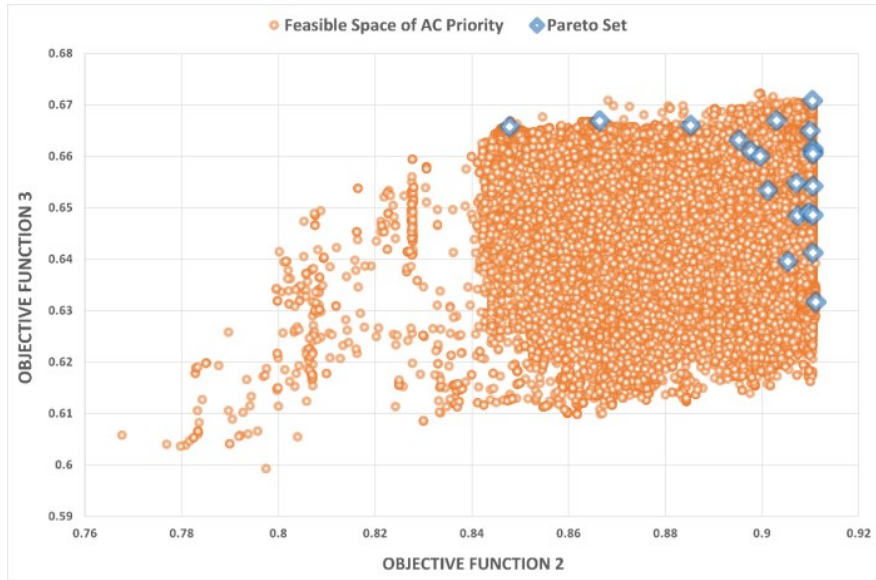

Fig. 13. The profile of feasible solutions under the 21 scenarios and the Pareto set for the two objective functions under the AC priority operation mode.

To demonstrate the validity of the optimization results, the convergence plots of the new fitness function $F^{\prime}$ are shown in Figure 14 for the DC priority operation mode and Figure 15 for the AC priority operation mode. The profiles of the Pareto sets in Figures 12 and 13 basically trace the upper-right boudary of the feasible region (which is the superposition of all the feasible solutions during the GA process) since both objectives are to be maximized. The Pareto frontier of the AC priority mode exhibits two lines (upper and right) along which small changes in one objective function come with large changes in the other. Interestingly, in the DC priority mode, the Pareto optimal solutions appear to be clustered in two regions, one with $f_{2} \sim 0.86$ and the other with $f_{2} \sim 0.82$. It is understandable that the former case corresponds to higher $f_{3}$ since increasing the capability of meeting DC demand will reduce that of meeting AC demand. The cause of this phenomenon is subject to follow-up studies. 


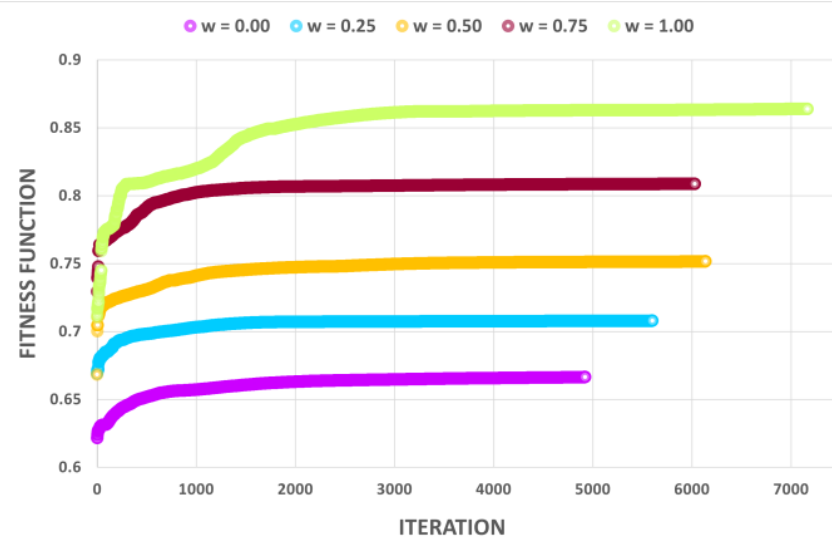

Fig. 14. The convergence of the values of the fitness function in five scenarios under the DC priority operation mode.

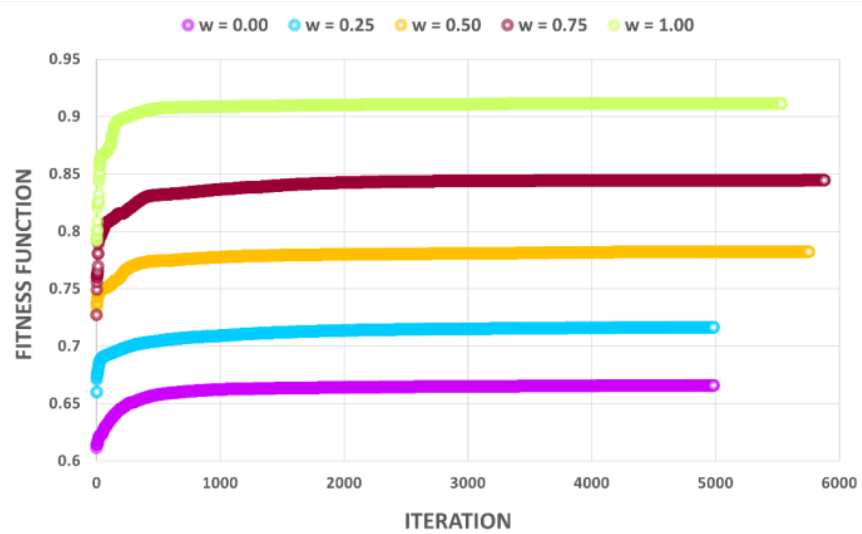

Fig. 15. The convergence of the values of the fitness function in five scenarios under the AC priority operation mode.

\section{CONCLUSION}

A multi-objective optimization framework is demonstrated for the design of a new form of BESS. Deployed with RES, the BESS comprises both conventional stationary batteries to meet intermittent demands from grid services and mobile batteries transported to meet continuous DC demand from EVCSs. The developed simulation model provides quantitative evidence that combining stationary and mobile applications can increase the profitability of BESS investment and reduce the curtailment of RES, while promoting electrical transportation. Two operation priorities (DC and $A C$ ) and scenarios with different weights on performance indices are simulated and compared, which will be valuable for future demonstration project planning. Further, Pareto frontier of a reduced-dimensional optimization problem is obtained to show the trade-off between two design objectives. This work lays the foundation for more in-depth studies such as improved multi-objective optimization techniques. Future work can also include the logistics systems modeling in the mobile BESS operation and financial analysis.

\section{REFERENCES}

[1] G. A. Barzegkar-Ntovom et al., "Assessing the viability of battery energy storage systems coupled with photovoltaics under a pure selfconsumption scheme," Renewable Energy, vol. 152, pp. 1302-1309, Jun. 2020, doi: 10.1016/j.renene.2020.01.061.
[2] Y. Liu, X. Wu, J. Du, Z. Song, and G. Wu, "Optimal sizing of a windenergy storage system considering battery life," Renewable Energy, vol. 147, pp. 2470-2483, Mar. 2020, doi: 10.1016/j.renene.2019.09.123.

[3] L. Xie et al., "Wind Integration in Power Systems: Operational Challenges and Possible Solutions," Proceedings of the IEEE, vol. 99, no. 1, Art. no. 1, Jan. 2011, doi: 10.1109/JPROC.2010.2070051.

[4] S. Liu, Z. Bie, J. Lin, and X. Wang, "Curtailment of renewable energy in Northwest China and market-based solutions," Energy Policy, vol. 123, pp. 494-502, Dec. 2018, doi: 10.1016/j.enpol.2018.09.007.

[5] E. Nycander, L. Söder, J. Olauson, and R. Eriksson, "Curtailment analysis for the Nordic power system considering transmission capacity, inertia limits and generation flexibility," Renewable Energy, vol. 152, pp. 942-960, Jun. 2020, doi: 10.1016/j.renene.2020.01.059.

[6] H. S. Hayajneh, S. Bashetty, M. N. B. Salim, and X. Zhang, "TechnoEconomic Analysis of a Battery Energy Storage System with Combined Stationary and Mobile Applications," in 2018 IEEE Conference on Technologies for Sustainability (SusTech), Nov. 2018, pp. 1-6. doi: 10.1109/SusTech.2018.8671332.

[7] "Wind Power Generation - an overview | ScienceDirect Topics," Jul. 02, 2019. https://www.sciencedirect.com/topics/engineering/windpower-generation (accessed Jul. 02, 2019).

[8] "Wind energy in Europe in 2018," WindEurope, Feb. 21, 2019. https://windeurope.org/about-wind/statistics/european/wind-energyin-europe-in-2018/ (accessed Jul. 02, 2019).

[9] daryl herzmann akrherz@iastate.edu, "Iowa Environmental Mesonet." https://mesonet.agron.iastate.edu/ (accessed Oct. 02, 2020).

[10] R. Hemmati, H. Mehrjerdi, and M. Bornapour, "Hybrid hydrogenbattery storage to smooth solar energy volatility and energy arbitrage considering uncertain electrical-thermal loads," Renewable Energy, vol. 154, pp. 1180-1187, Jul. 2020, doi: 10.1016/j.renene.2020.03.092.

[11] K. Bassett, R. Carriveau, and D. S.-K. Ting, "Energy arbitrage and market opportunities for energy storage facilities in Ontario," Journal of Energy Storage, vol. 20, pp. 478-484, Dec. 2018, doi: 10.1016/j.est.2018.10.015.

[12] Y. Xu and C. Singh, "Adequacy and Economy Analysis of Distribution Systems Integrated with Electric Energy Storage and Renewable Energy Resources," IEEE Transactions on Power Systems, vol. 27, no. 4, Art. no. 4, Nov. 2012, doi: 10.1109/TPWRS.2012.2186830.

[13] M. Quashie, C. Marnay, F. Bouffard, and G. Joós, "Optimal planning of microgrid power and operating reserve capacity," Applied Energy, vol. 210, pp. 1229-1236, Jan. 2018, doi: 10.1016/j.apenergy.2017.08.015.

[14] A. Berrada and K. Loudiyi, "Chapter 4 - Gravity Energy Storage Applications," in Gravity Energy Storage, A. Berrada and K. Loudiyi, Eds. Elsevier, 2019, pp. 75-103. doi: 10.1016/B978-0-12-8167175.00004-9.

[15] D. Zhu and Y. A. Zhang, "Optimal Coordinated Control of Multiple Battery Energy Storage Systems for Primary Frequency Regulation," IEEE Transactions on Power Systems, vol. 34, no. 1, Art. no. 1, Jan. 2019, doi: 10.1109/TPWRS.2018.2868504.

[16] J. Boyle, T. Littler, and A. Foley, "Battery energy storage system stateof-charge management to ensure availability of frequency regulating services from wind farms," Renewable Energy, vol. 160, pp. 11191135, Nov. 2020, doi: 10.1016/j.renene.2020.06.025.

[17] S. Karrari, M. Noe, and J. Geisbuesch, "High-speed Flywheel Energy Storage System (FESS) for Voltage and Frequency Support in Low Voltage Distribution Networks," in 2018 IEEE 3rd International Conference on Intelligent Energy and Power Systems (IEPS), Sep. 2018, pp. 176-182. doi: 10.1109/IEPS.2018.8559521.

[18] C. Li et al., "Method for the Energy Storage Configuration of Wind Power Plants with Energy Storage Systems used for Black-Start," Energies, vol. 11, no. 12, Art. no. 12, Dec. 2018, doi: $10.3390 /$ en 11123394

[19] U. G. Knight and U. G. Knight, Power Systems in Emergencies: From Contingency Planning to Crisis Management. Wiley, 2001.

[20] G. He, D. Zhang, X. Pi, Q. Chen, S. Kar, and J. Whitacre, "Spatiotemporal Arbitrage of Large-Scale Portable Energy Storage for Grid Congestion Relief," arXiv:1811.09924 [cs, math], Nov. 2018, Accessed: Jul. 10, 2019. [Online]. Available: http://arxiv.org/abs/1811.09924

[21] H. Khani, M. R. Dadash Zadeh, and A. H. Hajimiragha, "Transmission Congestion Relief Using Privately Owned Large-Scale Energy Storage Systems in a Competitive Electricity Market," IEEE Transactions on 
Power Systems, vol. 31, no. 2, pp. 1449-1458, Mar. 2016, doi: 10.1109/TPWRS.2015.2414937.

[22] "EIA - U.S. Battery Storage Market Trends," Jul. 10, 2019. https://www.eia.gov/analysis/studies/electricity/batterystorage/ (accessed Jul. 10, 2019).

[23] "Grid-connected battery energy storage systems market to reach 23.4 GW by 2022," Power Technology | Energy News and Market Analysis, May 15, 2018. https://www.power-technology.com/comment/gridconnected-battery-energy-storage-systems-market-reach-23-4-gw2022/ (accessed Jul. 10, 2019).

[24] S. Ferrey, "Efficiency in the Regulatory Crucible: Navigating 21st Century 'Smart' Technology and Power," JOURNAL OF ENERGY, p. 32, 2012.

[25] H. S. Hayajneh, M. Lainfiesta, and X. Zhang, "Three Birds One Stone: A Solution to Maximize Renewable Generation, Incentivize Battery Deployment, and Promote Green Transportation," in 2020 IEEE Power Energy Society Innovative Smart Grid Technologies Conference (ISGT), Feb. 2020, pp. 1-5. doi: 10.1109/ISGT45199.2020.9087710.

[26] A. Stephan, B. Battke, M. D. Beuse, J. H. Clausdeinken, and T. S. Schmidt, "Limiting the public cost of stationary battery deployment by combining applications," Nature Energy, vol. 1, no. 7, Art. no. 7, Jul. 2016, doi: 10.1038/nenergy.2016.79.

[27] R. T. Doucette and M. D. McCulloch, "Modeling the prospects of plugin hybrid electric vehicles to reduce $\mathrm{CO} 2$ emissions," Applied Energy, vol. 88, no. 7, Art. no. 7, Jul. 2011, doi: 10.1016/j.apenergy.2011.01.045.

[28] Zeming Jiang, Hao Tian, M. J. Beshir, R. Sibagatullin, and A. Mazloomzadeh, "Statistical analysis of Electric Vehicles charging, station usage and impact on the grid," in 2016 IEEE Power Energy Society Innovative Smart Grid Technologies Conference (ISGT), Sep. 2016, pp. 1-5. doi: 10.1109/ISGT.2016.7781228.

[29] M. Ban, M. Shahidehpour, J. Yu, and Z. Li, “A Cyber-Physical Energy Management System for Optimal Sizing and Operation of Networked Nanogrids With Battery Swapping Stations," IEEE Transactions on Sustainable Energy, vol. 10, no. 1, pp. 491-502, Jan. 2019, doi: 10.1109/TSTE.2017.2788056.

[30] X. Tan, G. Qu, B. Sun, N. Li, and D. H. K. Tsang, "Optimal Scheduling of Battery Charging Station Serving Electric Vehicles Based on Battery Swapping," IEEE Transactions on Smart Grid, vol. 10, no. 2, pp. 13721384, Mar. 2019, doi: 10.1109/TSG.2017.2764484.

[31] S. Wang, L. Yu, L. Wu, Y. Dong, and H. Wang, "An Improved Differential Evolution Algorithm for Optimal Location of Battery Swapping Stations Considering Multi-Type Electric Vehicle Scale Evolution," IEEE Access, vol. 7, pp. 73020-73035, 2019, doi: 10.1109/ACCESS.2019.2919507.

[32] G. Pan, W. Gu, Y. Lu, H. Qiu, S. Lu, and S. Yao, "Optimal Planning for Electricity-Hydrogen Integrated Energy System Considering Power to Hydrogen and Heat and Seasonal Storage," IEEE Transactions on Sustainable Energy, vol. 11, no. 4, pp. 2662-2676, Oct. 2020, doi: 10.1109/TSTE.2020.2970078.

[33] G. Taljan, C. Canizares, M. Fowler, and G. Verbic, "The Feasibility of Hydrogen Storage for Mixed Wind-Nuclear Power Plants," IEEE Transactions on Power Systems, vol. 23, no. 3, pp. 1507-1518, Aug. 2008, doi: 10.1109/TPWRS.2008.922579.

[34] L. Wang and H. Li, "Maximum Fuel Economy-Oriented Power Management Design for a Fuel Cell Vehicle Using Battery and Ultracapacitor," IEEE Transactions on Industry Applications, vol. 46, no. 3, pp. 1011-1020, May 2010, doi: 10.1109/TIA.2010.2045097.

[35] A. F. Burke, "Batteries and Ultracapacitors for Electric, Hybrid, and Fuel Cell Vehicles," Proceedings of the IEEE, vol. 95, no. 4, pp. 806820, Apr. 2007, doi: 10.1109/JPROC.2007.892490.

[36] P. Denholm, Y. Sun, and T. Mai, "An Introduction to Grid Services: Concepts, Technical Requirements, and Provision from Wind," p. 52.

[37] B. J. Kirby, "FREQUENCY REGULATION BASICS AND TRENDS," p. 32.

[38] H. S. Hayajneh, M. N. B. Salim, S. Bashetty, and X. Zhang, "Optimal Planning of Battery-Powered Electric Vehicle Charging Station Networks," in 2019 IEEE Green Technologies Conference (GreenTech), Apr. 2019, pp. 1-4. doi: 10.1109/GreenTech.2019.8767139.

[39] C. Shen, Q. Xiao, and Y. Zhang, "High-efficiency design method of LLC resonant converter for PHEV battery chargers (based on timedomain model)," IET Electrical Systems in Transportation, vol. 10, no. 3, pp. 234-242, 2020, doi: 10.1049/iet-est.2019.0092.
[40] "ERCOT-MERM Report_Final Draft-10.12.2018.pdf." Accessed: Oct. 03, 2020. [Online]. Available: http://www.ercot.com/content/wcm/lists/143980/10.12.2018_ERCOT _MERM_Report_Final_Draft.pdf

\section{Nomenclature}

$\begin{array}{ll}\boldsymbol{A C} & \text { Alternative Current } \\ \boldsymbol{B E} \boldsymbol{E S} & \text { Battery Energy Storage System } \\ \boldsymbol{C} \boldsymbol{F} & \text { Cash Flow } \\ \boldsymbol{C} \boldsymbol{V} & \text { Control Variable } \\ \boldsymbol{D C} & \text { Direct Current } \\ \boldsymbol{E C} \boldsymbol{A} & \text { End-Consumer Arbitrage } \\ \boldsymbol{E} \boldsymbol{F} & \text { Efficiency Factor } \\ \boldsymbol{E I} \boldsymbol{A} & \text { Energy } \\ \boldsymbol{E} \boldsymbol{V} & \text { Electric Vehicle } \\ \boldsymbol{E} \boldsymbol{V C S} & \text { Electric Vehicle Charging Station } \\ \boldsymbol{F R} & \text { Frequency Regulation } \\ \boldsymbol{G A} & \text { Genetic Algorithm } \\ \boldsymbol{G H} \boldsymbol{G} & \text { Greenhouse Gas } \\ \boldsymbol{I A} & \text { Investment Attractiveness } \\ \boldsymbol{O M} & \text { Operation and Maintenance } \\ \boldsymbol{P I} & \text { Profitability Index } \\ \boldsymbol{R E} \boldsymbol{S} & \text { Renewable Energy Source } \\ \boldsymbol{S O C} & \text { State of Charge } \\ \boldsymbol{U} \boldsymbol{G} & \text { Utility Grid } \\ \boldsymbol{V G} & \text { Value Generated }\end{array}$

